\title{
Signaltransduktion
}

\section{Zyklisches di-AMP - ein essenzielles Signalmolekül mit vielen Facetten}

JÖRG STÜLKE

INSTITUT FÜR MIKROBIOLOGIE UND GENETIK, UNIVERSITÄT GÖTTINGEN

\section{Many bacteria form the second messenger nucleotide cyclic di-AMP (c-di-AMP). This molecule is essential for many of the bacteria that produce it due to its central role in the control of potassium and osmolyte homeostasis. Moreover, under conditions of potassium starvation, a c-di-AMP target protein can trigger (p)ppGpp accumula- tion and thus a global reprogramming of cellular activities. Moreover, c-di-AMP formed by pathogens binds the human STING protein and triggers thus an immune response.}

DOI: $10.1007 / \mathrm{s} 12268-021-1565-9$ (C) Der Autor 2021

Alle Lebewesen müssen sich an plötzliche Veränderungen der Umweltbedingungen anpassen. Für die Wahrnehmung und Übertragung von Signalen steht ihnen ein großes Repertoire regulatorischer Mechanismen zur Verfügung. In Bakterien spielen Signalnukleotide, die Second Messenger, die als Antwort auf einen bestimmten Stimulus gebildet werden, eine ganz besondere Rolle. Das Paradebeispiel dieser Signalmoleküle ist das zyklische AMP, welches in Enterobakterien die C-Katabolitenrepression kontrolliert. Daneben gibt es auch eine Reihe zyklischer Di- und Trinukleotide, die in vielen Bakterien als Botenstoffe gebildet werden. Das zyklische di-GMP spielt in vielen Bakterien eine Schlüsselrolle bei der Wahl zwischen einem motilen und einem sessilen Lebensstil, also zwischen Bewegung und Biofilmbildung. Bei der Strukturanalyse eines Proteins, das in Bacillus subtilis die Integrität des DNA überwacht, fanden Gregor Witte und Kollegen vom Biozentrum München, dass dieses Protein ein bisher unbekanntes Molekül enthält, das sie als zyklisches di-AMP (c-di-AMP) identifizierten. Darüber hinaus konnten sie zeigen, dass dieses Protein, DisA, das c-di-AMP selbst produziert [1]. Inzwischen wissen wir, dass DisA der Prototyp für mehrere Proteinfamilien in Archaeen und Bakterien ist, die alle eine konservierte c-di-AMPsynthetisierende Domäne enthalten [2]. Während unter den Archaeen die c-di-AMP-Bildung im Wesentlichen auf die Euryarchaeota beschränkt ist, besitzen sehr viele Bakterien die Enzyme zur Bildung dieses Signalmoleküls. Eine wichtige Ausnahme sind dabei die Proteobakterien, von denen nur die Deltaproteobakterien c-di-AMP bilden.

Drei Beobachtungen rückten das c-di-AMP bald in den Fokus des Interesses:

- Dieses Molekül ist für viele Bakterien, die es bilden, essenziell, d. h. sie sind ohne C-di-AMP nicht lebensfähig [2].

- Die Suche nach Resistenzmechanismen gegenüber $\beta$-Lactam-Antibiotika ergab für viele Gram-positive Krankheitserreger, wie Staphylococcus aureus und Listeria monocytogenes, dass erhöhte Konzentrationen von c-di-AMP zur Resistenz gegenüber diesen Antibiotika beitragen, während reduzierte c-di-AMP-Mengen zu erhöhter Sensitivität führen [3].

- Das C-di-AMP kann von Krankheitserregern ausgeschieden werden und durch Bindung an den Interferon-Regulator STING eine Antwort des Immunsystems stimulieren [4].

Tab. 1: Bindepartner von c-di-AMP in Bacillus subtilis.

\begin{tabular}{|lllll|}
\hline Target & Art des Targets & Funktion & Konservierung & Effekt der Bindung \\
\hline DarA & Protein & Signaltransduktion & Ja & $?$ \\
\hline DarB & Protein & Signaltransduktion, Kontrolle von RelA & Partiell & Keine Bindung an RelA \\
\hline KimA & Protein & Kaliumaufnahme & Ja & Inhibition der Aktivität \\
\hline c-di-AMP Riboswitch $($ kimA $)$ & RNA & Kontrolle der kimA-Expression & Nein & Abbruch der Transkription \\
\hline KtrA & Protein & Kaliumaufnahme & Ja & Inhibition der Aktivität \\
\hline c-di-AMP Riboswitch $($ ktrAB) & RNA & Kontrolle der ktrAB-Expression & Nein & Abbruch der Transkription \\
\hline KtrC & Protein & Kaliumaufnahme & Ja & Inhibition der Aktivität \\
\hline CpaA & Protein & Kaliumexport & Partiell & Aktivierung des Kaliumexports \\
\hline KhtT & Protein & Kaliumexport & Partiell & Aktivierung des Kaliumexports \\
\hline OpuCA & Protein & Aufnahme kompatibler Solute & Ja & Inhibition der Aktivität \\
\hline MgtE & Protein & Magnesiumaufnahme & Ja & $?$ \\
\hline PycA & Protein & Stoffwechsel, Pyruvatcarboxylase & Ja & Inhibition der Aktivität \\
\hline
\end{tabular}




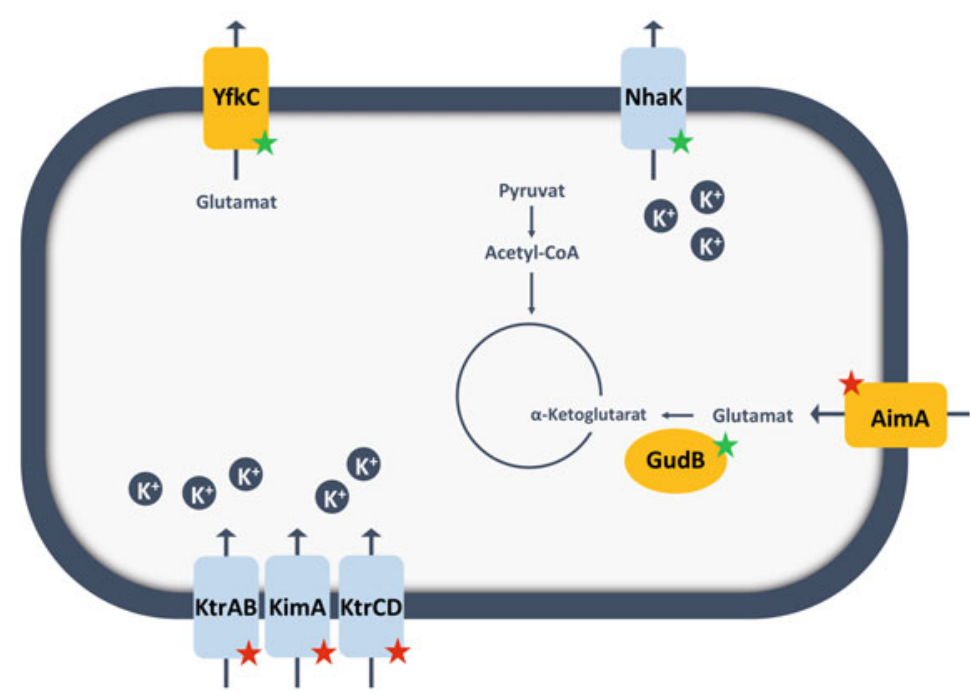

Abb. 1: Anpassung eines c-di-AMP-freien Stamms an das Wachstum in Gegenwart von Glutamat und Kalium. Die Mutationen aktivieren den Export von Kalium bzw. Glutamat, sie hemmen die Aufnahme der beiden Ionen oder stimulieren den Abbau von Glutamat. Grüner Stern: aktivierende Mutation; roter Stern: inaktivierende Mutation (modifiziert aus [11]).

In den vergangenen Jahren ist die Forschung zum Verständnis der Funktion(en) des c-di-AMP sehr schnell vorangeschritten, maßgeblich unterstützt durch das Schwerpunktprogramm der DFG zur Regulation durch bakterielle Second Messenger.

\section{c-di-AMP bindet Protein- und RNA-Moleküle}

Die spannendste Frage ist sicher, warum das c-di-AMP so wichtig ist für die Bakterien, die es produzieren. Zwei Wege wurden beschritten, um diese Frage zu beantworten: Um einen Einblick in die Funktion des c-di-AMP zu erhalten, wurden Moleküle in der Zelle identifiziert, an die es bindet. In einem komplementären Ansatz wurde versucht, Bedingungen zu finden, unter denen die Bakterien auch ohne c-di-AMP lebensfähig sind. Die Suche nach Bindungspartnern ergab, dass das c-di-AMP sowohl an Proteine als auch an RNA-Moleküle binden kann (Tab. 1, [2 ,5, 6]). Sehr viele Bindepartner des Signalmoleküls sind an der Kontrolle der intrazellulären Kaliumkonzentration beteiligt. Dabei handelt es sich um verschiedene Familien von Proteinen, die Kalium in die Zelle aufnehmen oder es ausschleusen können [2 ,5, 7]. In B. subtilis gibt es z. B. drei Kaliumaufnahmesysteme, die alle c-di-AMP binden können und durch diese Bindung inhibiert werden. Zwei dieser Transporter (KtrAB und KtrCD) gehören zur Trk-Familie, während der dritte (KimA) der Kup-Familie angehört. Obwohl zwischen diesen Proteinfamilien keine Ähnlichkeit besteht, haben beide im Verlauf der Evolution die Fähigkeit erworben, c-di-AMP zu binden und werden durch diese Bindung inhi- biert. Im Gegensatz dazu werden Kaliumexporter (in B. subtilis CpaA und KhtUT) durch c-di-AMP aktiviert. Diese Exporter enthalten eine Domäne, die auch in den Trk-Aufnahmesystemen vorhanden ist. Je nach Aktivität kann die Bindung von c-di-AMP an ähnliche Domänen also gegensätzliche Auswirkungen haben. Neben der Aktivität der Kaliumtransporter wird auch deren Expression durch c-di-AMP kontrolliert. In $L$. monocytogenes und $S$. aureus gibt es den hochaffinen KdpABC-Kaliumtransporter, dessen Expression durch das KdpDE-Zweikomponentensystem aktiviert wird. Die Sensorkinase KdpD bindet das c-di-AMP und wird dadurch wiederum inaktiviert, sodass bei hohen intrazellulären c-di-AMP-Konzentrationen das $k d p A B C$-Operon nicht exprimiert wird.

In vielen Bakterien stehen Gene für Kaliumtransporter unter der Kontrolle eines konservierten Riboschalters (der ydaORiboswitch). Dieses RNA-Molekül bindet C-di-AMP und führt in dieser Form zur Abschaltung der Transkription der kontrollierten Gene. In B. subtilis sind zwei Kopien dieses Riboswitches vorhanden, vor dem ktrAB-Operon und vor dem kimA-Gen (früher ydaO genannt) [7, 8]. In der B. cereus-Gruppe ist zusätzlich zu den Kaliumtransportern der Trk- und Kup-Familien auch das Kdp-System vorhanden. Hier wird es allerdings nicht durch das KdpDE-Zweikomponentensystem reguliert, sondern durch eine zusätzliche Kopie des c-di-AMP-bindenden Riboschalters. C-di-AMP ist damit das einzige bekannte Botenmolekül, das einen biologischen Prozess - nämlich die Kaliumaufnahme kontrolliert, indem es sowohl an die Proteine als auch an die für sie codierenden RNAMoleküle bindet.

Inzwischen haben mehrere Untersuchungen gezeigt, dass c-di-AMP als Reaktion auf die Verfügbarkeit von Kalium gebildet wird $[2,7]$. Ist viel Kalium vorhanden, kann das c-di-AMP durch seine Bindung an Transporter und Regulatoren die weitere Aufnahme verhindern und durch die Bindung an die Exporter sogar die Ausschleusung des Kaliums, das für die Zellen zwar essenziell, aber in zu hohen Konzentrationen auch toxisch ist, aktivieren. Damit ist das c-di-AMP ein wichtiger Faktor in der Kontrolle der Kaliumhomöostase in vielen Gram-positiven Bakterien [2].

Die Aufnahme von Kalium ist die erste Antwort auf hohe Salzkonzentrationen. Daher überrascht es nicht, dass das c-di-AMP auch die Anpassung an die osmotischen Verhältnisse beeinflusst. Viele osmoprotektive Substanzen sind bei unkontrollierter Aufnahme für die Zellen toxisch und c-di-AMP bindet in einigen Bakterien, z. B. B. subtilis, L. monocytogenes und $S$. aureus, an die ATPbindende Untereinheit eines ABC-Transporters für die Aufnahme von osmoprotektiven Substanzen, um so deren Aktivität zu inhibieren. In Milchsäurebakterien reguliert der Repressor BusR die Expression von Aufnahmesystemen für osmoprotektive Substanzen. Dieser Repressor enthält die gleiche regulatorische c-di-AMP-bindende Domäne, die auch in einigen Kaliumimportern und -exportern vorhanden ist und c-di-AMP wirkt als Korepressor für BusR, d. h. in Gegenwart von C-di-AMP werden die Osmotransporter nicht exprimiert [2, 6]. Das Vorhandensein dieser konservierten Domäne in funktionell ganz unterschiedlichen Proteinen ist ein schönes Beispiel für das Plug-and-Play-Prinzip der Evolution, bei dem immer wieder verschiedene Komponenten sinnvoll zusammengeführt werden können - in diesem Fall, um die Regulation durch c-di-AMP zu erreichen.

\section{c-di-AMP ist essenziell, weil es die Kalium- und Ionenhomöostase reguliert}

Ein zweiter Ansatz zum Verständnis der Essenzialität von c-di-AMP ist die Charakterisierung von Bedingungen bzw. Suppressormutationen, die Wachstum auch ohne das Molekül ermöglichen. So zeigte sich, dass C-di-AMP zwar für das Wachstum auf Komplexmedien, nicht aber auf Minimalmedien essenziell ist [9]. Da in Suppressormutanten auf Komplexmedium häufig die Aufnahme von kompatiblen Soluten betroffen ist, 
scheint der Schutz vor deren Toxizität eine zentrale Funktion des c-di-AMP zu sein [2,3, $9,10]$. Für B. subtilis sind in Abwesenheit von c-di-AMP Glutamat und Kalium auch beim Wachstum auf Minimalmedien toxisch. Suppressormutanten, die in Gegenwart erhöhter Kaliumkonzentrationen isoliert werden, enthalten immer eine Mutation, die zum verstärkten Kaliumexport führt - d. h., Kalium kann so entgiftet werden [7, 11]. Wenn Glutamat im Medium vorhanden ist, treten immer mehrere Suppressormutationen auf, die sowohl die Kalium- als auch die Glutamathomöostase betreffen (Abb. 1). Diese beiden Ionen sind die häufigsten positiv und negativ geladenen Ionen in jeder lebenden Zelle, und neben einer Reihe weiterer wichtiger Funktionen sind beide auch für die Anpassung an Osmostress essenziell. In $B$. subtilis führt die Verfügbarkeit von Glutamat dazu, dass ein normalerweise niedrigaffiner Kaliumtransporter ein hochaffines Aufnahmesystem wird. B. subtilis kann in Gegenwart von Glutamat nur dann ohne c-di-AMP leben, wenn die intrazellulären Konzentrationen von sowohl Glutamat als auch Kalium reduziert werden (durch verringerte Aufnahme, Export der Ionen oder im Fall von Glutamat, indem der Abbau aktiviert wird) [11]. Auch beim Wachstum auf Komplexmedium ist die Toxizität des Kaliums das Hauptproblem. Da der Kaliumtransport durch die protonenmotorische Kraft angetrieben wird, helfen auch Bedingungen (anaerobes Wachstum) oder Mutationen, die die Atmungskette betreffen, bei der Anpassung an Komplexmedium [11]. In $B$. subtilis spielen darüber hinaus zwei kleine c-di-AMP-bindende Proteine, die lediglich der Signaltransduktion dienen, eine wichtige Rolle in der Abwesenheit von c-di-AMP: Suppressormutanten eines C-di-AMP-freien Stamms konnten auf Komplexmedium nur dann isoliert werden, wenn eines dieser Signaltransduktionsproteine fehlt und darüber hinaus die Selektion unter anaeroben Bedingungen erfolgte [11]. Dies heißt, dass diese Signaltransduktionsproteine in Abwesenheit von c-di-AMP toxisch für die Zellen sind. Diese Beobachtung ist eine gute Grundlage zur Aufklärung der Funktion(en) dieser bisher wenig charakterisierten Proteine.

\section{Das Signaltransduktionsprotein DarB löst bei Kaliummangel die stringente Antwort aus}

Wie bereits erwähnt, hat Kalium viele wichtige Funktionen in der Zelle und ist mit gro-
Bem Abstand das wichtigste Kation. Eine dieser Funktionen ist die Translation, weil Kaliumionen essenziell für die Integrität des Ribosoms und die Architektur der wesentlichen funktionellen Regionen der Ribosomen sind. Ein Mangel an Kalium führt damit zu den gleichen Problemen bei der Translation wie ein Mangel an Aminosäuren, die in Proteine eingebaut werden können. Bei der Suche nach Interaktionspartnern für das Signaltransduktionsprotein DarB tauchte die Alarmonsynthetase RelA auf und es zeigte sich, dass DarB nur bei Kaliummangel (wenn kein c-di-AMP vorhanden ist) an RelA bindet und dadurch dann die (p)ppGpp-Synthese stimuliert. Wenn Kalium vorhanden ist, wird c-di-AMP gebildet und der C-di-AMP-DarB-Komplex kann nicht an RelA binden $[12,13]$. Somit gibt es einen Mechanismus, mit dem Kaliummangel über das DarB-Protein eine stringente Antwort auslöst. Da Kaliummangel ebenso wie das Fehlen einzelner Aminosäuren die Translation unmöglich macht, ist diese Reaktion für die Zelle sehr hilfreich.

\section{Offene Fragen}

Damit bleibt zu klären, welche Funktion das andere c-di-AMP-bindende Signaltransduktionsprotein, DarA, hat und ob die DarA- und DarB-Proteine in An- und Abwesenheit von C-di-AMP unterschiedliche Funktionen haben. Auch die genaue Rolle von c-di-AMP bei der Kontrolle der Zellwandsynthese bleibt weiter zu untersuchen. Da das c-di-AMP für viele Pathogene essenziell ist, könnten die Diadenylatzyklasen, die es synthetisieren, auch ein attraktives Target für die Suche nach neuen Antibiotika sein.

\section{Danksagung}

Ich danke vielen Mitarbeitern und Kollegen für die langjährige Zusammenarbeit zu diesem Thema, vor allem Christina Herzberg, Jan Gundlach, Larissa Krüger und Felix Mehne sowie Heike Bähre, Fabian Commichau, Ralf Ficner, Annette Garbe und Volkhard Kaever. Ein besonderer Dank geht an Regine Hengge für die Organisation des DFGSchwerpunktprogramms SPP1879, in dessen Rahme die hier vorgestellten Arbeiten gefördert werden.

\section{Literatur}

[1] Witte G, Hartung S, Büttner K et al. (2008) Structural biochemistry of a bacterial checkpoint protein reveals diadenylate cyclase activity regulated by DNA recombination intermediates. Mol Cell 30: 167-178
[2] Stülke J, Krüger L (2020) Cyclic di-AMP signaling in bacteria. Annu Rev Microbiol 74: 159-179

[3] Commichau FM, Gibhardt J, Halbedel S et al. (2018) A delicate connection: c-di-AMP affects cell integrity by controlling osmolyte transport. Trends Microbiol 26: 175-185 [4] Archer KA, Durack J, Portnoy DA (2014) STING dependent type I IFN production inhibits cell-mediated immunity to Listeria monocytogenes. PLoS Pathog 10: e1003861

[5] Corrigan RM, Campeotto I, Jeganathan T et al. (2013) Systematic identification of conserved bacterial c-di-AMP receptor proteins. Proc Natl Acad Sci U S A 110: 9084-9089 [6] He J, Yin W, Galperin MY et al. (2020) Cyclic di-AMP, a second messenger of primary importance: tertiary structures and binding mechanisms. Nucleic Acids Res 48 . 2807-2829

[7] Gundlach J, Herzberg C, Kaever V et al. (2017) Control of potassium homeostasis is an essential function of the second messenger cyclic di-AMP in Bacillus subtilis. Sci Signal 10: eaal3011

[8] Nelson JW, Sudarsan N, Furukawa K et al. (2013) Riboswitches in eubacteria sense the second messenger c-di-AMP. Nat Chem Biol 9: 834-839

[9] Whiteley AT, Pollock AJ, Portnoy DA (2015) The PAMP $\mathrm{c}$-di-AMP is essential for Listeria monocytogenesgrowth in rich but not in minimal media due to a toxic increase in $(\mathrm{p})$ ppGpp. Cell Host Microbe 17: 788-798

[10] Zeden MS, Schuster CF, Bowman L et al. (2018) Cyclic di-adenosine monophosphate (c-di-AMP) is required for osmotic regulation in Staphyloccus aureus but dispensable for viability in anaerobic conditions. J Biol Chem 293: 31803200

[11] Krüger L, Herzberg C, Rath H et al. (2021) Essentiality of c-di-AMP in Bacillus subtilis: bypassing mutations converge in potassium and glutamate homeostasis. PLoS Genet 17: e1009092

[12] Peterson BN, Young MKM, Luo S et al. (p)ppGpp and c-di-AMP homeostasis is controlled by CbpB in Listeria monocytogenes. mBio 11: e01625-20

[13] Krüger L, Herzberg C, Wicke D et al (2021) A meet-up of two second messengers: The c-di-AMP receptor DarB controls (p)ppGpp synthesis in Bacillus subtilis. Nat Commun 12: 1210

Funding note: Open Access funding enabled and organized by Projekt DEAL. Open Access: Dieser Artikel wird unter der Creative Commons Namensnennu 4.0 International Lizenz veröfenticht, welche die Nutzung, Vervielfältigung, erlaubt, sofern Sie den/die ursprünglichen Autor(en) und die Quelle ordnungsgemäß nennen, einen Link zur Creative Commons Lizenz beifügen un angeben, ob Änderungen vorgenommen wurden. Die in diesem Artikel angeben, ob Änderungen vorgenommen wurden. Die in diesem Artikel
enthaltenen Bilder und sonstiges Drittmaterial unterliegen ebenfalls der genannten Creative Commons Lizenz, sofern sich aus der Abbildungslegende nichts anderes ergibt. Sofern das betreffende Material nicht unter der genannten Creative Commons Lizenz steht und die betreffende Handlung nicht nach gesetzlichen Vorschriften erlaubt ist, ist für die oben aufgeführten Weiterverwendungen des Materials die Einwilligung des jeweiligen Rechteinhabers einzuholen. Weitere Details zur Lizenz entnehmen Sie bitte der

Korrespondenzadresse:

Prof. Dr. Jörg Stülke

Abt. Allgemeine Mikrobiologie

Institut für Mikrobiologie und Genetik

Georg-August-Universität Göttingen

Grisebachstraße 8

D-37077 Göttingen

jstuelk@gwdg.de

\begin{tabular}{|c|c|}
\hline AUTOR & \\
\hline $10 \bar{c}$ & $\begin{array}{l}\text { Jörg Stülke } \\
\text { Jahrgang } 1964 . \\
\text { 1985-1990 Biologiestudium } \\
\text { an der Universität Greifswald, } \\
\text { dort } 1994 \text { Promotion. } \\
\text { 1994-1996 Postdoc am } \\
\text { Pasteur-Institut Paris, Frank- } \\
\text { reich. 1996-2003 Arbeits- } \\
\text { gruppenleiter an der Univer- } \\
\text { sität Erlangen. } 2000 \text { Habilita- } \\
\text { tion. Seit 2003 Professor für } \\
\text { Mikrobiologie an der Univer- } \\
\text { sität Göttingen. }\end{array}$ \\
\hline
\end{tabular}

